\title{
Alterações bioquímicas e hepáticas em ratos submetidos à uma dieta hiperlipídica/hiperenergética ${ }^{1}$
}

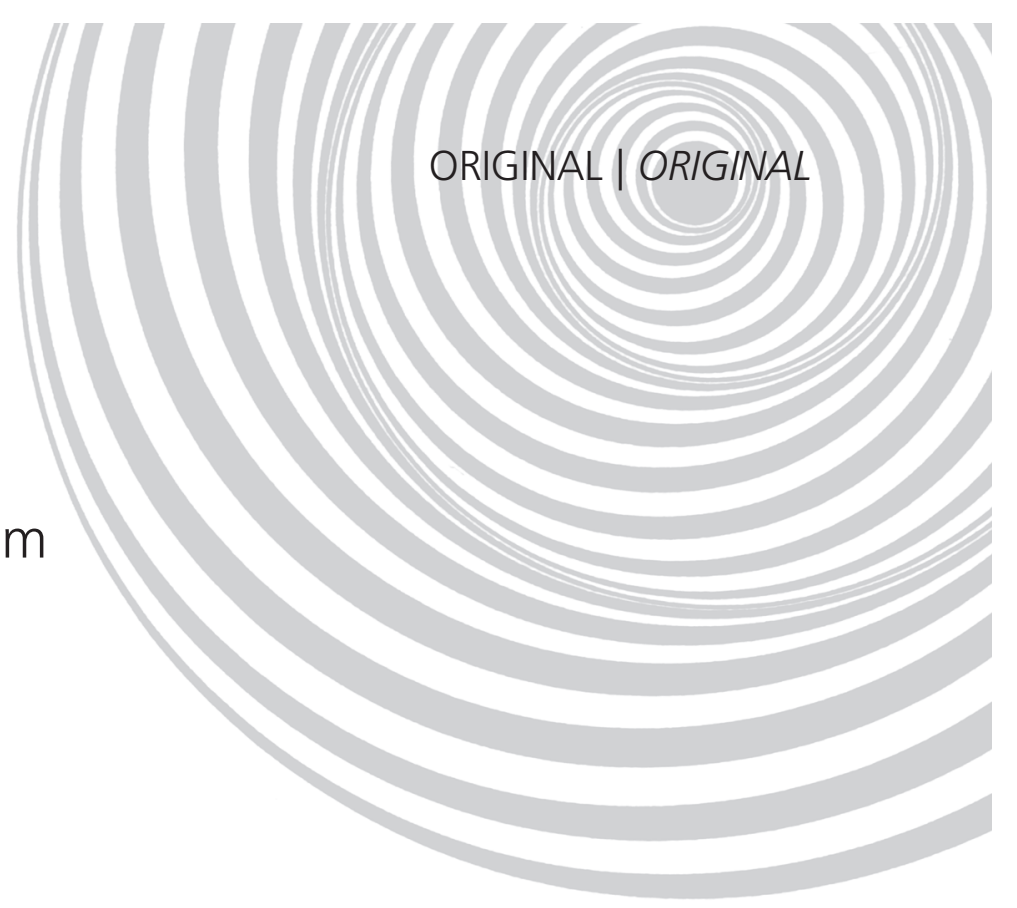

\author{
Hepatic biochemical changes in rats \\ submitted to a high-fat/high-energy diet
}

Leandro Pereira de MOURA²

Rodrigo Augusto DALIA²

Michel Barbosa de ARAÚjO²

Amanda Christine da Silva SPONTON²

José Rodrigo PAULI ${ }^{3}$

Rodrigo Ferreira de MOURA²

Maria Alice Rostom de MELLO²

RE S U M O

\section{Objetivo}

O presente estudo teve como objetivo analisar as alterações bioquímicas hepáticas decorrentes da administração de uma dieta hiperlipídica/hiperenergética em ratos.

\section{Métodos}

Foram utilizados 20 ratos (Wistar) com 90 dias de idade divididos em dois grupos, grupo-controle constituída por ratos eutróficos alimentados com dieta comercial para roedores e grupo-dieta constituída por ratos submetidos a uma dieta hiperlipídica/hiperenergética semi purificada feita com 35\% de gordura sendo $31 \%$ de origem animal a qual possui 39\% de gordura saturada e $4 \%$ de origem vegetal (óleo de soja). Os animais do grupo-controle foram mantidos com dieta comercial Purina ${ }^{\circledR}$ e o grupo-dieta com uma dieta hiperlipídica/ hiperenergética constituída por 35\% de gordura. Após 60 dias de administração de uma dieta hiperlipídica/ hiperenergética, analisou-se massa corporal, sensibilidade à insulina, concentração sérica de glicose, insulina e ácidos graxos livres e medida do nível de triglicerídeos, lipídeos totais e atividade lipogênica hepática.

\section{Resultados}

O grupo-dieta apresentou maior massa corporal e resistência à insulina. No sangue não foram encontradas diferenças entre os grupos para os níveis de glicose. Foi evidenciada maior concentração de insulina e de

\footnotetext{
1 Apoio: Fundação de Amparo à Pesquisa do Estado de São Paulo (Processo: 2010/12718-5), Fundação para o Desenvolvimento da Universidade Estadual de São Paulo, Coordenação de Aperfeiçoamento de Pessoal de Nível Superior e Conselho Nacional de Desenvolvimento Científico e Tecnológico.

2 Universidade Estadual Paulista Júlio de Mesquita Filho, Instituto de Biociências, Departamento de Educação Física. Av. 24 A, 1515, Bela Vista, 13506-900, Rio Claro, SP, Brasil. Correspondência para/Correspondence to: LP MOURA. E-mail: <leandropereiram@hotmail.com>.

${ }^{3}$ Universidade Estadual de Campinas, Faculdade de Ciências Aplicadas, Curso de Ciências do Esporte. Limeira, SP, Brasil.
} 
686 | LP MOURA et al.

ácidos graxos livres no soro para o grupo-dieta. No fígado o nível de lipídeos totais, triglicerídeos e taxa lipogênica foram superiores às do grupo-controle.

\section{Conclusão}

Portanto, nossos achados demonstram que dois meses de ingestão de dieta hiperlipídica/hiperenergética por ratos adultos eleva o peso corporal, ácidos graxos livres hepáticos, diminui a sensibilidade à insulina, demostrando sinais típicos de doença hepática gordurosa não-alcoólica.

Termos de indexação: Dieta hiperlipídica. Doença hepática gordurosa não alcoólica. Resistência à insulina. Sobrepeso.

\section{A B S TR A C T}

\section{Objective}

The present study analyzed the biochemical and hepatic changes in adult rats fed a high-fat diet for two months.

\section{Methods}

Twenty Wistar rats 90 days old were divided into two groups, a control group consisting of normal weight rats fed a commercial rat chow and a diet group consisting of normal weight rats submitted to a semipurified high-fat, high-energy diet. The animals in control group were kept on a commercial Purina ${ }^{\circledR}$ chow and those in diet group on a high-fat/high-energy diet containing 35\% fat, of which 31\% were from animal source (39\% saturated fat) and 4\% were from vegetable source (soybean oil). After 60 days of this experimental diet, the following were assessed: body weight, insulin sensitivity, blood glucose, serum insulin and free fatty acids, triglycerides, total lipids and hepatic lipogenic activity.

\section{Results}

Diet group presented higher body mass and insulin resistance. Blood glucose did not differ between the groups. A higher level of serum insulin and free fatty acids were found in diet group. Total lipids, triglycerides and lipogenic rate were also higher in group $D$.

\section{Conclusion}

Therefore, the present findings demonstrate that two months of a high-fat/high-energy diet increases the body weight and hepatic free fatty acids and decreases insulin sensitivity of adult rats, typical signs of non-alcoholic fatty liver disease.

Indexing terms: Diet, high-fat. Non-alcoholic fatty liver disease. Insulin resistance. Overweight.

\section{N T R O D U ÇÃ O}

Atualmente, os países em desenvolvimento passam por uma transição nutricional, caracterizada pela diminuição da prevalência da desnutrição e pelo aumento da obesidade ${ }^{1}$. Desde a década passada, a obesidade tem sido considerada a mais importante desordem nutricional nos países desenvolvidos e em desenvolvimento, acometendo mais de $10,0 \%$ da população mun-

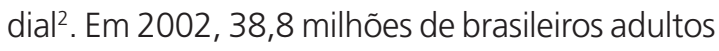
estavam acima do peso; hoje essa taxa chega a 54,2\% da população do País, e cresce acentuadamente, indiferentemente de sexo e idade ${ }^{3}$. Definida como doença desde 1985 e caracterizada por acúmulo excessivo de gordura, a obesidade é um problema de saúde pública mundial que pode desencadear a morte ${ }^{4,5}$.

O consumo de gordura aumentou em todo o mundo e ficou claro que, ao lado da alta quantidade energética dos alimentos atualmente consumidos, certas composições dietéticas que associam o consumo excessivo de gorduras saturadas e sal e o consumo insuficiente de frutas e hortaliças aumentam a adiposidade corporal e apresentam forte associação com doenças crônicas degenerativas não transmissíveis ${ }^{6}$.

O comprometimento da qualidade de vida de uma pessoa obesa decorre das várias doenças associadas a essa condição, como: câncer ${ }^{7}$, hiper- 
tensão arterial, hipercolesterolemia, hiperlipidemia, doenças coronarianas, diabetes Mellitus ${ }^{8}$, invalidez ${ }^{9}$ e Doença Hepática Gordurosa não Alcoólica $(\mathrm{DHGNA})^{10}$, que cresce concomitante à incidência de obesidade ${ }^{11}$.

O acúmulo de gordura no fígado é decorrente, principalmente, da elevação dos Ácidos Graxos Livres (AGL) na corrente sanguínea ${ }^{12}$. O AGL em excesso, caso não seja oxidado ou transportado para a circulação em forma de lipoproteínas de baixa densidade, pode ser sintetizado em triacilgliceróis e depositado no fígado, podendo dar início à $\mathrm{DHGN}^{12}$ e reduzir o cleareance hepático de insulina, aumentando assim a produção hepática de glicose, fatores chaves no processo de Resistência à Insulina $(\mathrm{RI})^{13}$. O aumento do $\mathrm{AGL}$ circulante vai atuar, também, nos receptores de insulina, debilitando seu funcionamento nos diversos tecidos e com isso levando à hiperglicemia e hiperinsulinemia ${ }^{14}$. O fígado gorduroso, caso não diagnosticado e não tratado, pode levar a um aumento dos tecidos conjuntivos do fígado, conduzindo o órgão à falência ${ }^{10}$. Diante do exposto e das limitações éticas das pesquisas com humanos, como o envolvimento de técnicas invasivas, o presente trabalho teve como objetivo investigar as alterações bioquímicas e do metabolismo hepático decorrentes da oferta de uma dieta hiperlipídica/hiperenergética em ratos.

\section{MÉ T O D O S}

Para o desenvolvimento deste trabalho, foram utilizados 30 ratos Wistar jovens (90 dias de idade e massa corporal de Média $(M)=400,18$, Desvio-Padrão $(\mathrm{DP})=41,88 \mathrm{~g}$ no início do experimento). Os animais, provenientes do Biotério Central da Universidade Estadual Paulista Júlio de Mesquita Filho (Unesp), Botucatu (SP) foram mantidos no Biotério do Departamento de Educação Física do Instituto de Biologia (IB) da Unesp de Rio Claro (SP). Os ratos foram tratados com ração/ dieta e água ad libitum e mantidos em gaiolas plásticas coletivas (quatro ratos em cada gaiola) à temperatura ambiente de $\mathrm{M}=22, \mathrm{DP}=1^{\circ} \mathrm{C} \mathrm{e}$ fotoperíodo de 12 horas de claro e 12 de escuro.
O experimento foi realizado de acordo com a legislação brasileira sobre o uso científico de animais (Lei no 11.794, de 8 de outubro de 2008). O Protocolo foi aprovado pela Comissão de Ética no Uso de Animal (CEUA), do Instituto de Biociências da UNESP, Campus de Rio Claro, Protocolo $n^{\circ} 2010 / 4573$.

O período experimental foi de 60 dias. Os animais do grupo-controle foram mantidos com dieta equilibrada (PURINA ${ }^{\circledR}, 3.028 \mathrm{kcal} / \mathrm{kg}$ ), e os animais do grupo experimental foram mantidos com dieta desequilibrada semipurificada hiperlipídica/hiperenergética $(5.350 \mathrm{kcal} / \mathrm{kg})$, composta por ( $\mathrm{g} \%$ ): amido de milho=26,4; caseína=14; dextrina $=8,8$; sacarose $=5,6$; lipídeos $=35$ (sendo $4 \%$ de origem vegetal (óleo de soja Sadia ${ }^{\circledR}$ ) e 31\% de origem animal (gordura suína Sadia $\left.{ }^{\circledR}\right)$ ) microcelulose $=5$, mistura de sais minerais $=3,5$; mistura de vitaminas 1,0; L-cistina $=1,8$ e bitartarato de colina $=2,5$.

A composição detalhada das misturas de sais minerais e de vitaminas empregadas na confecção da dieta hiperlipídica/hiperenergética acha-se descrita em Reeves et al. ${ }^{15}$.

Os grupos experimentais formados por ratos Wistar com 90 dias de idade foram divididos em dois grupos conforme administração da dieta: grupo-controle $(\mathrm{C})$, constituído por 10 ratos tratados com ração comercial Purina ${ }^{\circledR}$; e grupo-dieta (D), constituído por 10 ratos tratados com dieta semipurificada hiperlipídica/hiperenergética ao longo de todo período experimental.

Todos os animais tiveram massa corporal registrada no final do experimento por meio de balança semianalítica (Marte Balance ${ }^{\circledR}$ ).

A sensibilidade à insulina foi avaliada pelo Teste de Tolerância à Insulina (TTI). Para isso, foi realizada uma primeira coleta de sangue através de corte na extremidade da cauda do animal (tempo zero). Em seguida, uma solução de insulina cristalina "LILLY U 40", na dose de 30mU/100g de peso corporal, foi administrada via subcutânea. Amostras de sangue foram coletadas após 30, 60 e 120 minutos com capilares heparinizados e 
calibrados para $25 \mu \mathrm{L}$, visando à determinação das concentrações de glicose, utilizando kits comerciais $\left(\right.$ Laborlab $\left.^{\circledR}\right)$. Um único corte na extremidade da cauda do animal foi suficiente para a coleta de todas as amostras sanguíneas. A taxa de remoção de glicose (Kitt) expressa em \%/minuto foi calculada pela fórmula $\left(0,0693 / t_{12}\right) \times 100$. A glicose sanguínea (t/2) foi calculada pela curva de análise dos mínimos quadrados dos teores de glicose no sangue nos momentos após a administração de insulina em que a glicose sérica decaiu ${ }^{16}$.

No final do experimento, 48 horas depois da última avaliação in vivo, os animais na condição alimentada e em repouso foram sacrificados depois de serem anestesiados com gás carbônico; seu sangue foi coletado, centrifugado a 3.000 rpm por 15 minutos, e, através das amostras do soro, foram realizadas as análises de glicose (Kit Laborlab ${ }^{\circledR}$ ), insulina por método de radioimunoensaio $^{17}$ e $\mathrm{AGL}^{18}$. Alíquotas do fígado foram retiradas para determinação de lipídeos totais e triglicerídeos. As amostras foram colocadas em tubos com TritonX-100 a 0,1\%, e, em seguida, foram homogeneizadas em Polytron ${ }^{\circledR}$ por 20 segundos em velocidade máxima; após esse procedimento, as amostras foram centrifugadas a 4.000 rpm por um período de 10 minutos. O sobrenadante foi extraído para a determinação dos lipídeos totais ${ }^{19} \mathrm{e}$ triglicerídeos por espectrofotometria através de kit comercial (Laborlab $\left.{ }^{\circledR}\right)$.

Um lote de animais ( $n=5$ animais/grupo) que receberam o mesmo tratamento descrito anteriormente foi sacrificado 60 minutos após receberem administração intraperitoneal de $3 \mathrm{mCi}$ $3 \mathrm{H} 2 \mathrm{O}$. Alíquotas do tecido hepático foram extraídas e pesadas para determinação da taxa de lipogênese, utilizando-se água triciada, como previamente descrito por Robinson \& Williamson ${ }^{20}$. A taxa de síntese de lipídeos foi expressa em $\mu \mathrm{mol}$ de $3 \mathrm{H}_{2} \mathrm{O}$ incorporada em lipídeos por hora, por grama de tecido.

Os resultados foram expressos em média e desvio-padrão. O teste $t$ de Student para amostras independentes foi empregado para a comparação entre as médias do peso corporal no final do experimento, taxa de remoção de glicose sérica (Kitt), concentrações séricas de glicose, insulina e AGL e lipídeos totais e triglicerídeos hepáticos. 0 nível de significância estabelecido foi de p<0,05.

\section{RESULTADOS}

No término do período experimental, os animais do grupo $D$ tiveram sua massa corporal superior aos do grupo C (Figura 1A). A sensibi-

A
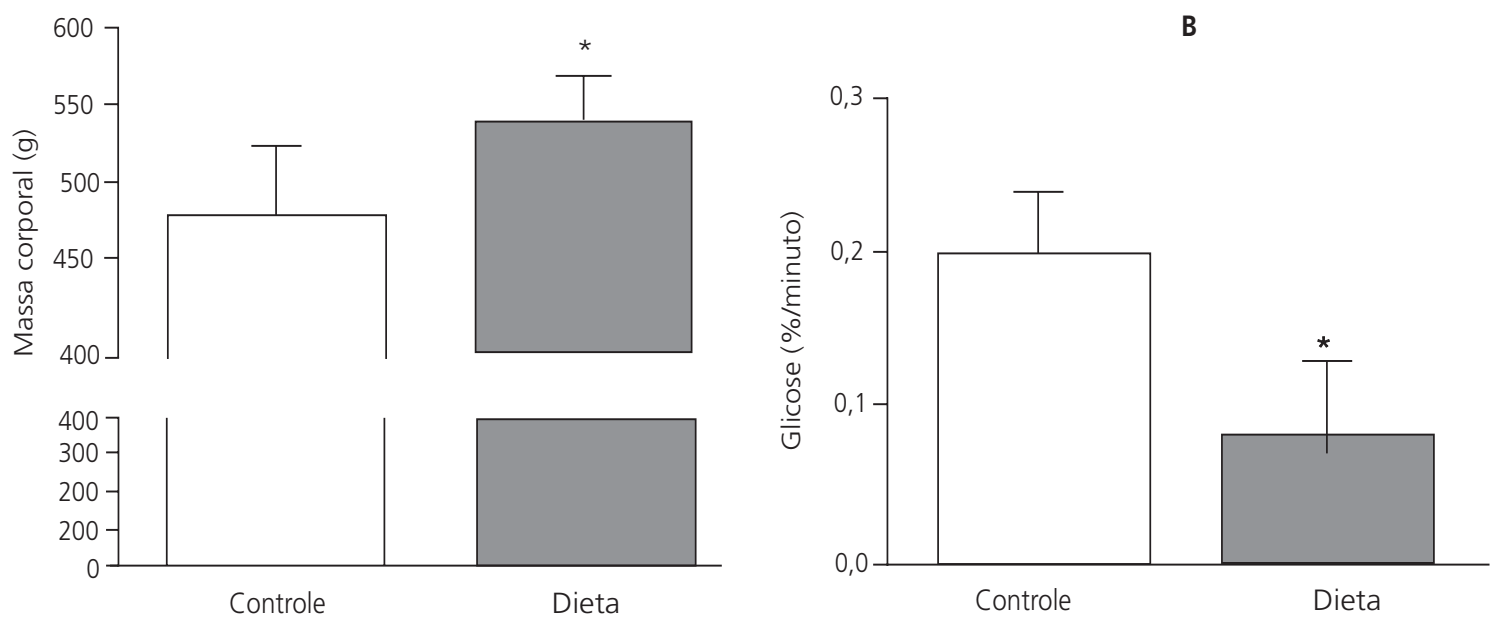

Figura 1. Massa corporal (A), teste de tolerância à insulina (taxa de remoção de glicose/minuto) (B) ao final de 60 dias de experimento. Resultados expressos com média e desvio-padrão ( $n=10 / g r u p o)$.

Nota: ${ }^{*}=$ diferente do grupo-controle (teste $t, p<0,05$ ). 
lidade periférica à insulina do grupo $D$ foi inferior quando comparada ao grupo C (Figura 1B).

As concentrações séricas de glicose não apresentaram diferenças entre os grupos estudados ao final do experimento (Figura 2A). Os níveis séricos de insulina e $A G L$ foram maiores para o grupo $D$ em relação ao grupo $C$ (Figura 2B e 2C).

A atividade lipogênica hepática foi superior para o grupo $D$ em relação ao $C$ ao final do experimento (Figura 3A). A quantidade de lipídeos totais hepáticos encontrada no grupo $D$ foi superior em relação à encontrada no grupo $C$ (Figura 3B). O acúmulo de gordura em forma de triglicerídeos no fígado foi superior para o grupo $D$ em relação ao grupo C (Figura 3C).

A

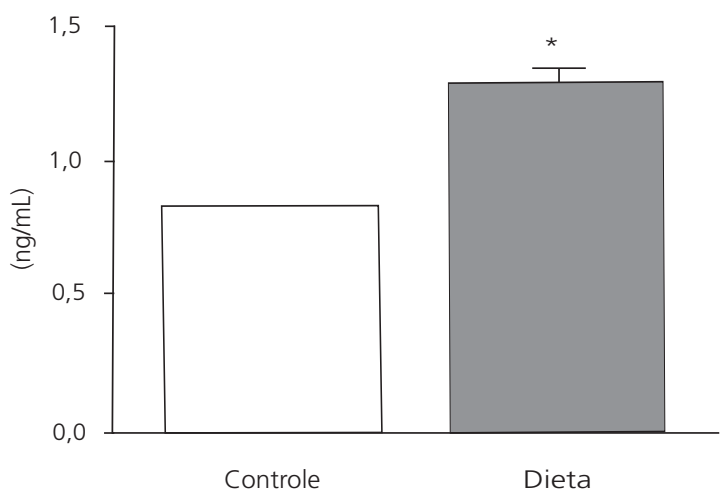

\section{I S C U S S Ã O}

Devido ao aumento no consumo de gordura em todo o mundo, faz-se necessária maior investigação das consequências do alto consumo de lípides, especificamente, para o fígado. De acordo com os dados aqui obtidos, ficou claro que o alto consumo de gordura leva a um quadro de obesidade, com aumento das concentrações de AGL séricas, que irá interferir tanto na sinalização da insulina, levando a RI, quanto contribuir para a síntese de triglicerídeos hepáticos, que conduz à DHGNA.

Desde 2003, existem mais de 30 modelos genéticos de obesidade descritos ${ }^{21}$. Contudo, outros procedimentos também vêm sendo usados

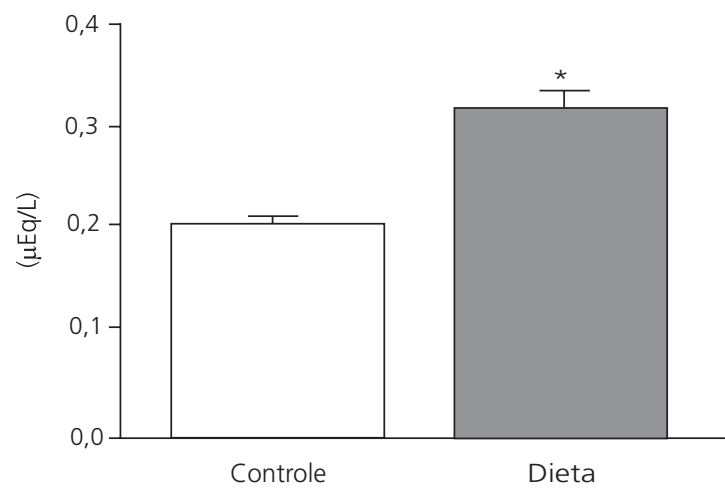

C

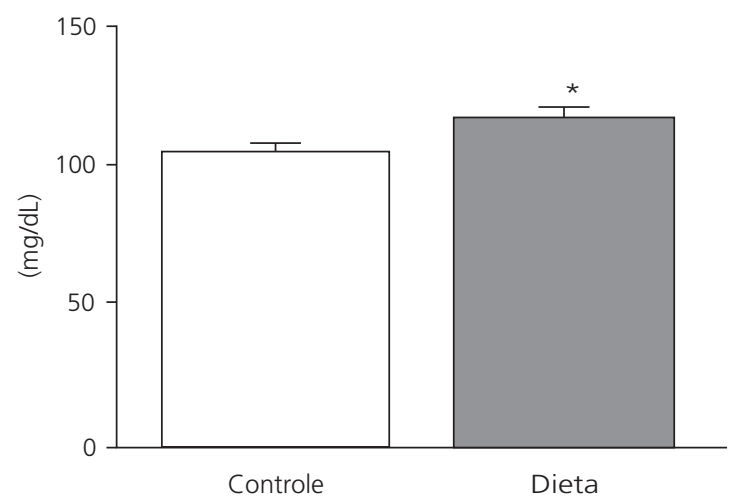

Figura 2. Glicose (A), insulina (B) e AGL (C) séricos ao final de 60 dias de experimento. Resultados expressos com média e desvio-padrão ( $n=10 /$ grupo)

Nota: ${ }^{*}=$ diferente do grupo-controle (teste $t, p<0,05$ ) 
690 | LP MOURA et al.

A

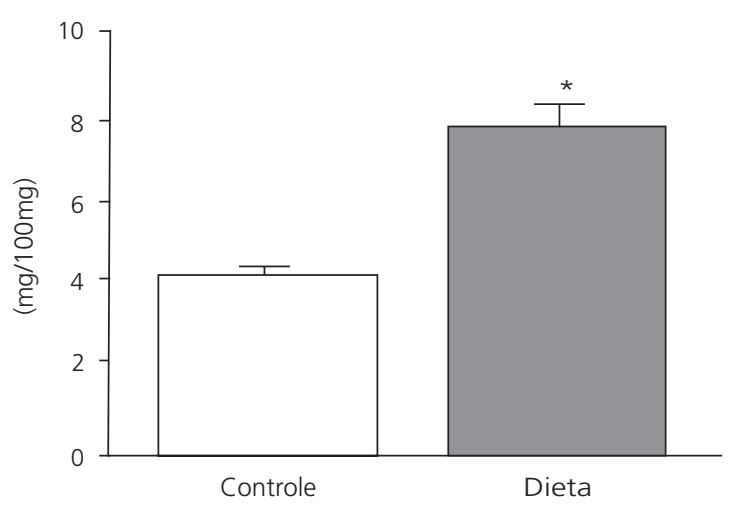

B

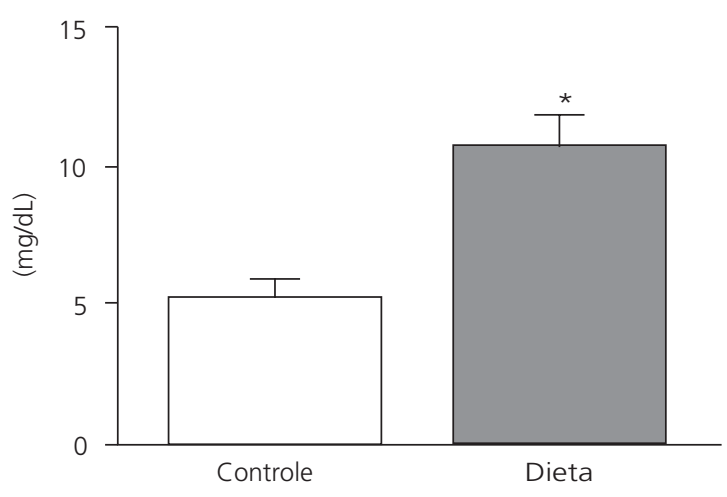

C

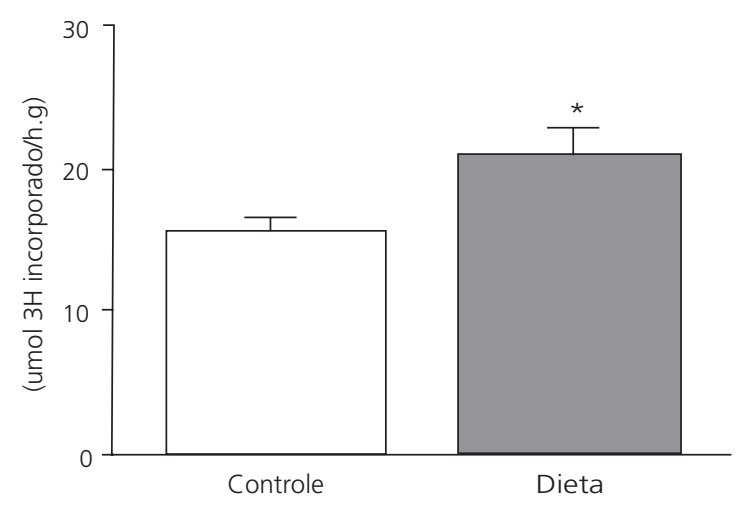

Figura 3. Lipogênese (5 animais/grupo) (A), lipídeos totais (B) e triglicerídeos (C) hepáticos ao final de 60 dias de experimento. Resultados expressos com média e desvio-padrão ( $n=10 /$ grupo).

Nota: * $=$ diferente do grupo-controle (teste $t, p<0,05)$.

para mimetizar a má alimentação do ser humano e, concomitantemente, o desenvolvimento da obesidade, como dieta hiperenergética ${ }^{22}$ ou indução neural da obesidade através do uso do Glutamato Monossódico (MSG), que destrói o núcleo arqueado do hipotálamo, responsável pela regulação complexa dos hormônios e neuropeptídios responsáveis pelo controle do peso em roedores ${ }^{23}$.

Muitas pesquisas têm sido feitas em torno do desequilíbrio nutricional das dietas para ratos. Com o intuito de mimetizar a alimentação desequilibrada humana e seus transtornos decorrentes, como resistência à insulina ou obesidade, temse obtido êxito através do uso de modelos experimentais com animais ${ }^{23}$. Na atualidade, uma estratégia de pesquisa bastante utilizada para o desenvolvimento da obesidade é a administração de uma dieta rica em lípides a roedores ${ }^{24}$.

A gordura como substrato energético oferece $9 \mathrm{kcal} / \mathrm{g}$ de energia, proporcionando um balanço positivo e, consequentemente, acúmulo em forma de triglicerídeos, caso ela não seja mobilizada para suprir a necessidade do organismo ${ }^{25}$. De acordo com alguns estudos, a grande oferta de energia unida à inatividade física auxilia o ganho de peso e acúmulo de gordura corporal, como visto no presente estudo, que corrobora a literatura ${ }^{7,24}$. Nesses estudos, ratos alimentados com dieta hiperlipídica (35\% de lipídeos) foram comparados a outros que receberam dieta equili- 
brada contendo $4 \%$ de gordura vegetal para as necessidades básicas do organismo. O ganho de peso dos animais alimentados com dieta hiperlipídica foi relativamente maior do que dos mantidos com dieta equilibrada 7,24 .

Os resultados do presente trabalho corroboram, ainda, uma revisão literária feita por Cesaretti \& Kohlmann Jr. ${ }^{26}$ e outros estudos experimentais em animais ${ }^{24,27}$ em que os autores mostraram que a utilização de dieta hiperlipídica/hiperenergética é o modelo experimental que mais se assemelha à $\mathrm{Rl}$ e à obesidade encontradas em seres humanos.

O aumento no consumo de gordura ou a incompleta inibição da enzima lípase, hormônio sensível decorrente da RI, faz com que os níveis de $A G L$ séricos aumentem ${ }^{28}$. Esses $A G L$ podem ser convertidos em triglicerídeos no fígado ${ }^{12}$. A RI foi definida por Himsworth \& Kerr' ${ }^{29}$ como diminuição da sensibilidade à insulina nos tecidos periféricos, ocasionando uma resposta glicêmica deficiente à insulina exógena, encontrada em pessoas obesas com diabetes Mellitus tipo 2 (DM2). Essa perda de sensibilidade ao hormônio está relacionada a um estado em que são necessárias concentrações elevadas de insulina para manter a homeostase glicêmica, levando ao quadro de hiperinsulinemia ${ }^{30}$.

A responsividade dos tecidos à insulina pode sofrer danos devido à elevação nas concentrações de $A G L$ no sangue. Os AGL desempenham importantes funções fisiológicas no músculo esquelético, coração, fígado e pâncreas, mas seu aumento compromete a via de sinalização da insulina, podendo induzir em indivíduos com DM2 o agravamento da resistência periférica e a queda da secreção de insulina após um período de hiperinsulinemia ${ }^{30}$. Esse quadro de DM2 com elevação de AGL reduz a captação de glicose pelos tecidos por comprometer a transmissão de sinal na via de sinalização da insulina, com consequente diminuição no número de GLUT-4 translocados para a membrana plasmática. Neste estudo ficou claro o sucesso da indução da obesidade e das alterações decorrentes, como hiperinsulinemia, dimi- nuição da sensibilidade à insulina e aumento das concentrações séricas de AGL.

A administração da dieta hiperlipídica gerou obesidade associada a aumento nas concentrações séricas de AGL, o que possivelmente prejudicou a via de sinalização da insulina, gerando um quadro de RI. Essas duas alterações estão fortemente relacionadas ao acúmulo de gordura no fígado ${ }^{31}$. O aumento de gordura circulante proporciona maior disponibilidade de gordura para o fígado e esta maior oferta associada a uma baixa taxa de catabolismo leva a um desequilíbrio entre absorção, síntese e exportação de gordura, gerando acúmulo de lípides nesse órgão e iniciando a DHGNA $^{12,30}$.

Doença hepática gordurosa não alcoólica é caracterizada principalmente pela presença de macrogotículas de gordura no hepatócito. $\mathrm{Na}$ ausência de processos inflamatórios, esse fenômeno é conhecido como esteatose hepática ${ }^{32}$. Uma forma segura de analisar a instalação da DHGNA é mensurar a quantidade de gordura armazenada no órgão (TG e lipídeos totais) e juntamente analisar a quantidade de gordura que está sendo sintetizada no próprio tecido (atividade lipogênica). Neste estudo, conseguimos combinar essas análises e verificar o total de gordura que foi armazenada e a quantidade que foi sintetizada em um curto espaço de tempo (uma hora). Podemos correlacionar esses achados com os de Oosterveer et al. ${ }^{33}$, que também analisaram a atividade lipogênica e encontraram, assim como neste estudo, a instalação da DHGNA através da administração de dieta rica em gordura.

\section{CONCLUSÃO}

Portanto, na luz desses achados, observa-se que a oferta de dieta hiperlipídica/hiperenergética a ratos adultos, no período de dois meses, induz aumento da massa corporal e da concentração de ácidos graxos livres no fígado e reduz a sensibilidade à insulina: sinais característicos da DHGNA. 
692 | LP MOURA et al.

\section{A G RADECIMENTOS}

Aos técnicos Clarice Sibuya e José Roberto Rodrigues, ao Professor Dennys Esper Correa Cintra, ao Conselho Nacional de Desenvolvimento Científico e Tecnológico e à Fundação de Amparo à Pesquisa do Estado de São Paulo, pelo suporte financeiro, e a Corn Products Brasil ${ }^{\circledR}$, pela doação do material da dieta usado no experimento.

\section{OLABORADORES}

Todos os autores colaboraram para o estudo na coleta, análise e discussão dos dados e na redação do manuscrito, sendo que todos leram e aprovaram a versão final.

\section{REFERÊ N CIAS}

1. Amuna P, Zotor FB. Epidemiological and nutrition transition in developing countries: impact on human health and development. Proc Nutr Soc. 2008; 67:82-90.

2. World Health Organization. Obesity: preventing and managing the global epidemic. Geneva: WHO; 1998. Report of a WHO Consultation on Obesity.

3. Instituto Brasileiro de Geografia e Estatística. POF 2008-2009: antropometria e estado nutricional de crianças, adolescentes e adultos no Brasil. Rio de Janeiro: IBGE; 2008.

4. Marti A, Marcos A, Martinez J. Obesity and immune function relationships. Obes Rev. 2001; 2(2):131-40.

5. Mendes MJFL, Alves JGB, Alves AV, Siqueira PP, Freire EFC. Associação de fatores de risco para doenças cardiovasculares em adolescentes e seus pais. Rev Bras Saúde Matern Infant. 2006; 6:49-54.

6. World Health Organization. Diet, nutrition and the prevention of chronic diseases. Geneva: WHO; 2003. WHO Technical Report Series, 916.

7. Seth D, Garmo H, Wigertz A, Holmberg L, Hammar $\mathrm{N}$, Jungner $\mathrm{I}$, et al. Lipid profiles and the risk of endometrial cancer in the Swedish AMORIS study Int J Mol Epidemiol Genet. 2012; 3(2):122-33.

8. Guedes DP, Guedes JERD, Barbosa DS, Oliveira JA, Stanganelli LCR. Fatores de risco cardiovasculares em adolescentes: indicadores biológicos e comportamentais. Arq Bras Cardiol. 2006; 86:439-50.

9. Ezzati M, Lopez AD, Rodgers A, Van Der Hoorn S, Murray CJL. The comparative risk assessment collaborating group: selected major risk factors and global and regional burden of disease. Lancet. 2002; 360(9343):1347-60.

10. Utzschneider KM, Kahn SE. Review: the role of insulin resistance in nonalcoholic fatty liver disease. J Clin Endicrinol Metab. 2006; 91:4753-61.

11. Williams R. Global challenges in liver disease. Hepatology. 2006; 44:521-6.

12. Goldberg IJ, Ginsberg HN. Ins and outs modulating hepatic triglyceride and development of nonalcoholic fatty liver disease. Gastroenterology. 2006; 130(4):1343-46.

13. Carvalho MHC, Colaço AL, Fortes ZB. Citocinas, disfunção endotelial e resistência à insulina. Arq Bras Endocrinol Metab. 2006; 50(2):304-12.

14.Pratchayasakul W, Kerdphoo S, Petsophonsakul P, Pongchaidecha A, Chattipakorn N, Chattipakorn SC, et al. Effects of high-fat diet on insulin receptor function in rat hippocampus and the level of neuronal corticosterone. Life Sci. 2011; 88(13-4): 619-27.

15. Reeves PG, Nielsen FH, Fahey GC JR. AIN-93 purified diets for laboratory rodents: final report of the American Institute of Nutrition ad hoc writing committee on the reformulation of the AIN76Arodent diet. J Nutr. 1993; 123(11):1939-51.

16. Lundbaeck K. Intravenous glucose tolerance test as a tool in definition and diagnosis of diabetes Mellitus. Br Med J. 1962; 1(529):1507-13.

17. Herbert V, Lau KS, Gottlieb CW, Bleicher SJ. Coated Charcoals immunoassay of insulin. J Clin Endocrinol Metab. 1965; 25(10):1375-84.

18. Regow BJM, Cornelissen PJHC, Helder APR, Spijkers JBF, Weeber YMM. Specific determination of free fatty acid in plasm. Clin Acta Chim. 1971; 31(1): 187-95.

19. Nogueira DM, Strufaldi B, Hirata MH, Abdalla DSP, Hirata RDC. Métodos de bioquímica clínica: técnicas e interpretação. São Paulo: Pancast; 1990.

20. Robinson AM, Williamson DH. Control of glucose metabolism in isolated acini of the lactanting mammary gland of the rat: effects of oleat on glucose utilization and lipogenesis. Biochemistry J. 1978; 170(3):609-13.

21. Pereira LO, Francischi RP, Lancha Junior AH. Obesidade: hábitos nutricionais, sedentarismo e resistência à insulina. Arq Bras Endocrinol Metab. 2003; 47(2):111-27.

22. Lladó I, Rodríguez-Cuenca S, Pujol E, Monjo M, Estrany M, Roca P, et al. Gender effects on adrenergic receptor expression and lipolysis in white adipose tissue of rats. Obes Res. 2002; 10(4):296-305. 
23. Araújo GG, Araújo MB, D’Angelo RA, Manchado FB, Mota CSA, Ribeiro C, et al. Máxima Fase estável de lactato em ratos obesos de ambos os gêneros. Rev Bras Med Esporte. 2009; 15(1):46-9.

24. Ropelle ER, Pauli JR, Prada PO, Souza CT, Picardi PK, Faria MC, et al. Reversal of diet-induced insulin resistance with a single bout of exercise in the rat: the role of PTP1B and IRS-1 serine phosphorylation. J Physiol. 2006; 577(3):997-1007.

25. Flatt JP. Use and storage of carbohydrate and fat. Am J Clin Nutr. 1995; 61:952-9.

26. Cesaretti MRL, Kohlmann Jr O. Modelos experimentais de RI e obesidade. Arq Bras Endocrinol Metab. 2006; 50(2):90-197.

27. Ropelle ER, Pauli JR, Prada P, Cintra DE, Rocha GZ, Moraes JC, et al. Inhibition of hypothalamic Foxo1 expression reduced food intake in diet-induced obesity rats. J Physiol. 2009; 587(Pt10):2341-51.

28. Machado M, Cortez-Pinto H. Non-alcoholic fatty liver disease and insulin resistance. Eur J Gastroent Hepatol. 2005; 17(8):823-6.
29. Himsworth HP, Kerr RB. Insulin-sensitive and insulininsensitive types of diabetes mellitus. Clin Sci (London). 1939; 4:120-52.

30. Boden G, Shulman GI. Free fatty acids in obesity and type 2 diabetes: defining their role in the development of insulin resistance and $\beta$-cell dysfunction. Eur J Clin Invest. 2002; 32(3):14-23.

31. Postic C, Girard J. Contribution of de novo fatty acid synthesis to hepatic steatosis and insulin resistance: lessons from genetically engineered mice. J Clin Invest. 2008; 118(3):829-38.

32. Diehl AM. Nonalcoholic steatohepatitis. Semin Liver Dis. 1999; 19(2):221-9.

33. Oosterveer MH, Van Dijk TH, Tietge UJF, Boer T, Havinga R, Stellaard F, et al. High fat feeding induces hepatic fatty acid elongation in mice. PLoS ONE. 2009; 4(6):e6066. doi:10.1371/journal.pone. 0006066.
Recebido em: 8/2/2012

Versão final em: 3/7/2012

Aprovado em: 20/8/2012 
